\title{
The Coin Problem for Product Tests
}

\author{
CHIN HO LEE and EMANUELE VIOLA, Northeastern University
}

Let $X_{m, \varepsilon}$ be the distribution over $m$ bits $\left(X_{1}, \ldots, X_{m}\right)$, where the $X_{i}$ are independent and each $X_{i}$ equals 1 with probability $(1+\varepsilon) / 2$ and 0 with probability $(1-\varepsilon) / 2$. We consider the smallest value $\varepsilon^{*}$ of $\varepsilon$ such that the distributions $X_{m, \varepsilon}$ and $X_{m, 0}$ can be distinguished with constant advantage by a function $f:\{0,1\}^{m} \rightarrow S$, which is the product of $k$ functions $f_{1}, f_{2}, \ldots, f_{k}$ on disjoint inputs of $n$ bits, where each $f_{i}:\{0,1\}^{n} \rightarrow S$ and $m=n k$

We prove that $\varepsilon^{*}=\Theta(1 / \sqrt{n \log k})$ if $S=[-1,1]$, while $\varepsilon^{*}=\Theta(1 / \sqrt{n k})$ if $S$ is the set of unit-norm complex numbers.

CCS Concepts: • Mathematics of computing $\rightarrow$ Random number generation; • Theory of computation $\rightarrow$ Computational complexity and cryptography; Pseudorandomness and derandomization;

Additional Key Words and Phrases: Coin problem, product test

\section{ACM Reference format:}

Chin Ho Lee and Emanuele Viola. 2018. The Coin Problem for Product Tests. ACM Trans. Comput. Theory 10, 3, Article 14 (June 2018), 10 pages.

https://doi.org/10.1145/3201787

\section{INTRODUCTION}

Let $X_{m, \varepsilon}$ be the distribution over $m$ bits $\left(X_{1}, \ldots, X_{m}\right)$, where the $X_{i}$ are independent and each $X_{i}$ equals 1 with probability $(1+\varepsilon) / 2$ and 0 with probability $(1-\varepsilon) / 2$. The $\varepsilon$-coin problem is the problem of distinguishing the distributions $X_{m, \varepsilon}$ and $X_{m, 0}$ (note that the latter is the uniform distribution). A threshold function gives the best possible distinguishing advantage; specifically, it can distinguish the two distributions for $m=O\left(1 / \varepsilon^{2}\right)$, and this is asymptotically the lowest value of $m$ for every function. Thus the coin problem is therefore most interesting in computational models that cannot compute thresholds. The study of the coin problem goes back at least to the 1970 article by Hellman and Cover [14]. Since then, the coin problem has been extensively studied and has found applications in a striking variety of contexts, including computing majority and approximate majority using low-depth circuits by Ajtai [3] and Valiant [22], barriers to circuit lower bounds [19], pseudorandom generators [6], quantum computing [1,2], and multiparty computation [7].

Shaltiel and Viola [19] give an $\mathrm{AC}^{0}$ reduction from Majority to the coin problem, thereby obtaining negative results for the coin problem for bounded-depth circuits with various types of gates. Aaronson [1] and Cohen et al. [8] improve the parameters of the negative result for $\mathrm{AC}^{0}$.

This work is supported by National Science Foundation grant CCF-1319206.

Authors' addresses: C. H. Lee and E. Viola, 360 Huntington Av., \#202 WVH, Boston, MA 02115; emails: \{chlee, viola\}@ccs. neu.edu.

Permission to make digital or hard copies of all or part of this work for personal or classroom use is granted without fee provided that copies are not made or distributed for profit or commercial advantage and that copies bear this notice and the full citation on the first page. Copyrights for components of this work owned by others than ACM must be honored. Abstracting with credit is permitted. To copy otherwise, or republish, to post on servers or to redistribute to lists, requires prior specific permission and/or a fee. Request permissions from permissions@acm.org.

(C) 2018 ACM 1942-3454/2018/06-ART14 $\$ 15.00$

https://doi.org/10.1145/3201787 
Brody and Verbin [6] and Steinberger [21] study the $\varepsilon$-coin problem for small-width branching programs. A generalization of the problem is studied in Reference [8]. For constant width, References $[6,21]$ obtain tight bounds on $\varepsilon$. However, the case of larger widths remains open and looks very difficult.

In this work, we consider a special case of large-width branching programs that we call product tests. A product test is a function $f$ on $n k$ bits that equals the product of $k$ functions $f_{1}, f_{2}, \ldots, f_{k}$ on $k$ disjoint inputs of $n$ bits. We will consider $f_{i}$ with two different ranges: $[-1,1]$, which includes the special cases of $\{0,1\}$ and $\{-1,1\}$, and unit-norm complex numbers. We will settle the coin problem for both ranges. Extensions to the range of complex numbers have proved very useful. For example, Reference [10] derives a pseudorandom generator for halfspaces and a number of other tests considered in the literature from one that works for complex-valued products.

The model of product tests has been extensively studied in pseudorandomness [4, 5, 9-13, 15$18,23,24]$, at least in part with the hope that it will be useful to understand general branching programs. Thus it seems natural to study the coin problem for product tests.

First, we prove that if the $f_{i}$ have range $[-1,1]$, then a product test cannot solve the $\varepsilon$-coin problem for $\varepsilon=o(1 / \sqrt{n \log k})$.

THEOREM 1.1. Let $f:\{0,1\}^{n k} \rightarrow[-1,1]$ be a product of $k$ functions $f_{1}, \ldots, f_{k}:\{0,1\}^{n} \rightarrow$ $[-1,1]$, each on disjoint inputs of $n$ bits. Let $\alpha \in(0,1 / 4]$ and $\varepsilon=\alpha /(32 \sqrt{n \log (k / \alpha)})$. We have $\left|\mathrm{E}\left[f\left(X_{n k, 0}\right)\right]-\mathrm{E}\left[f\left(X_{n k, \varepsilon}\right)\right]\right| \leq O(\alpha \log (1 / \alpha))$.

We then show that Theorem 1.1 is tight, even for the range $\{0,1\}$.

THEOREM 1.2. For every $k \in\left[n^{2}, 2^{n}\right]$, there exists an $\varepsilon=O(1 / \sqrt{n \log k})$, and a function $f$ : $\{0,1\}^{n k} \rightarrow\{0,1\}$ that is a product of $k$ functions $f_{1}, \ldots, f_{k}:\{0,1\}^{n} \rightarrow\{0,1\}$, each on disjoint inputs of $n$ bits, such that $\operatorname{Pr}\left[f\left(X_{n k, 0}\right)=1\right]-\operatorname{Pr}\left[f\left(X_{n k, \varepsilon}\right)=1\right] \geq 1 / 100$.

To summarize, we have established that, for $S=[-1,1], \varepsilon^{*}=\Theta(1 / \sqrt{n \log k})$ is the smallest value of $\varepsilon$ such that the distributions $X_{m, \varepsilon}$ and $X_{m, 0}$ can be distinguished with constant advantage by a function $f:\{0,1\}^{m} \rightarrow S$, which is the product of $k$ functions $f_{1}, f_{2}, \ldots, f_{k}$ on disjoint inputs of $n$ bits, where each $f_{i}:\{0,1\}^{n} \rightarrow S$ and $m=n k$. For comparison, recall from earlier in the Introduction that for arbitrary Boolean functions on $n k$ bits, the corresponding value is $\varepsilon^{*}=\Theta(1 / \sqrt{n k}$ ) (that $\varepsilon^{*}=\Omega(1 / \sqrt{n k})$ also follows from Claim 4 in this article; and Claim 5 extends this to complexvalued functions).

Finally, we note that if the range is enlarged to the set $\mathbb{C}_{=1}:=\{z \in \mathbb{C}:|z|=1\}$ of complex numbers with unit norm, the picture changes completely and product tests are as powerful as arbitrary functions.

Claim 1. For every integer $k$ and $\varepsilon>0$, there exists $f:\{-1,1\}^{n k} \rightarrow \mathbb{C}_{=1}$, which is a product of $k$ functions $f_{1}, \ldots, f_{k}:\{0,1\}^{n} \rightarrow \mathbb{C}_{=1}$, each on disjoint inputs of $n$ bits, such that $\mid \mathrm{E}\left[f\left(X_{n k, 0}\right)\right]-$ $\mathrm{E}\left[f\left(X_{n k, \varepsilon}\right)\right] \mid \geq 1.5-e^{-\Omega\left(\varepsilon^{2} n k\right)}$.

Organization. We begin with some useful facts and definition in Section 2. Then, in Section 3, we prove Theorem 1.1. Finally, we prove Theorem 1.2 and Claim 1 in Section 4.

\section{PRELIMINARIES}

We use the notation $\pm \alpha$ in the right-hand side of an equality to indicate that the equality holds if we replace $\pm \alpha$ with $z$ for some $z$ such that $|z| \leq \alpha$. We use this notation multiple times, with the meaning that each occurrence is replaced by a possibly different $z$.

Throughout the article, all logarithms are in base 2, and we will use the following bounds. 
Claim 2 (Hoeffoding's Inequality). Let $X_{1}, \ldots, X_{n} \in[-1,1]$ be $n$ independent and identically distributed variables with $\mathrm{E}\left[X_{i}\right]=\mu$ for each $i$. We have

$$
\operatorname{Pr}\left[\left|\sum_{i=1}^{n} X_{i}-\mu n\right| \geq t\right] \leq 2 e^{-t^{2} / 2 n} .
$$

Claim 3 (Maclaurin's Inequality (CF. [20])). Let $z_{1}, \ldots, z_{k}$ be $k$ non-negative numbers. For any $i \in\{0, \ldots, k\}$, we have

$$
S_{i}\left(z_{1}, \ldots, z_{k}\right):=\sum_{S: \mid S=i} \prod_{j \in S} z_{j} \leq(e / i)^{i}\left(\sum_{j=1}^{k} z_{j}\right)^{i} .
$$

To get a sense of the inequality, note that if the $z_{i}$ 's equal to 1 , then it becomes the standard upper bound on the binomial coefficient $\left(\begin{array}{c}k \\ i\end{array}\right) \leq(e k / i)^{i}$.

Next we formally define our main distributions.

Definition 2.1. Let $X_{n, \varepsilon}=\left(X_{1}, \ldots, X_{n}\right)$ be the distribution over $n$ bits, where the $X_{i}$ 's are independent and each $X_{i}$ equals 1 with probability $(1+\varepsilon) / 2$ and 0 with probability $(1-\varepsilon) / 2$. Let $N_{n, \varepsilon}$ denote the sum of the $X_{i}$ 's.

Note that we have $\mathrm{E}\left[N_{n, \varepsilon}\right]=(1+\varepsilon) n / 2$.

\section{PROOF OF THEOREM 1.1}

We start with the following claim about a single function on $n$ bits. Note that in particular this claim implies the well-known fact that one function cannot solve the $\varepsilon$-coin problem for $\varepsilon=o(1 / \sqrt{n})$.

Claim 4. For every $\alpha \in[0,1]$ and $\beta \in(0,1 / 2]$, let $\varepsilon=\alpha /(32 \sqrt{n \log (1 / \beta)})$. For any function $f$ : $\{0,1\}^{n} \rightarrow[0,1]$, we have $\mathrm{E}\left[f\left(X_{n, \varepsilon}\right)\right]=\mathrm{E}\left[f\left(X_{n, 0}\right)\right](1 \pm \alpha) \pm \beta$.

Proof. For a string $x \in\{0,1\}^{n}$, we use $w t(x)$ to denote its Hamming weight. The high-level idea is that if $w t(x)$ is far from $n / 2$, then by Hoeffding's inequality we can bound above the expectation of both $f\left(X_{n, \varepsilon}\right)$ and $f\left(X_{n, 0}\right)$ by $\beta$. Otherwise, since the two probabilities of drawing $x$ differ by a multiplicative factor of $(1+\varepsilon)^{w t(x)}(1-\varepsilon)^{n-w t(x)}$, we can use the fact that $w t(x)$ is close to $n / 2$ to show that this factor is within $1 \pm \alpha$.

To proceed, let $T:=4 \sqrt{n \log (1 / \beta)}$. Let $S_{1}:=\{x:|w t(x)-n / 2| \leq T\}$ and $S_{2}:=\{x: \mid w t(x)-$ $n / 2 \mid>T\}$.

Since $\beta \leq 1 / 2$, we have $\log (1 / \beta) \geq 1$ and so

$$
\varepsilon n / 2=\alpha \sqrt{n} /(64 \sqrt{\log (1 / \beta)}) \leq \sqrt{n} / 64 \leq T / 2 .
$$

By Hoeffding's inequality,

$$
\begin{aligned}
\mathrm{E}\left[f\left(X_{n, \varepsilon}\right) \mathbb{1}\left(X_{n, \varepsilon} \in S_{2}\right)\right] & \leq \mathrm{E}\left[\mathbb{1}\left(X_{n, \varepsilon} \in S_{2}\right)\right] \\
& =\operatorname{Pr}\left[\left|N_{n, \varepsilon}-n / 2\right|>T\right] \\
& \leq \operatorname{Pr}\left[\left|N_{n, \varepsilon}-(1+\varepsilon) n / 2\right|>T-\varepsilon n / 2\right] \\
& =\operatorname{Pr}\left[\left|N_{n, \varepsilon}-\mathrm{E}\left[N_{n, \varepsilon}\right]\right|>T-\varepsilon n / 2\right] \\
& \leq \operatorname{Pr}\left[\left|N_{n, \varepsilon}-\mathrm{E}\left[N_{n, \varepsilon}\right]\right|>T / 2\right] \\
& \leq 2 e^{-2 \log (1 / \beta)} \leq 2 \beta^{2} \leq \beta,
\end{aligned}
$$

and the same bound holds for $\mathrm{E}\left[f\left(X_{n, 0}\right) \mathbb{1}\left(X_{n, 0} \in S_{2}\right)\right]$. So the two expectations differ by at most $\beta$ in absolute value. 
Now we show that $\mathrm{E}\left[f\left(X_{n, \varepsilon}\right) \mathbb{1}\left(X_{n, \varepsilon} \in S_{1}\right)\right]=\mathrm{E}\left[f\left(X_{n, 0}\right) \mathbb{1}\left(X_{n, 0} \in S_{1}\right)\right](1 \pm \alpha)$. We have

$$
\begin{aligned}
\mathrm{E}\left[f\left(X_{n, \varepsilon}\right) \mathbb{1}\left(X_{n, \varepsilon} \in S_{1}\right)\right] & =\sum_{x \in S_{1}} f(x) \operatorname{Pr}\left[X_{n, \varepsilon}=x\right] \\
& =2^{-n} \sum_{x \in S_{1}} f(x)(1+\varepsilon)^{w t(x)}(1-\varepsilon)^{n-w t(x)} \\
& =2^{-n} \sum_{x \in S_{1}} f(x)(1+\varepsilon)^{n / 2-(n / 2-w t(x))}(1-\varepsilon)^{n / 2+(n / 2-w t(x))} \\
& =2^{-n} \sum_{x \in S_{1}} f(x)\left(1-\varepsilon^{2}\right)^{n / 2}\left(\frac{1+\varepsilon}{1-\varepsilon}\right)^{w t(x)-n / 2} .
\end{aligned}
$$

Recall that $T=4 \sqrt{n \log (1 / \beta)}$ and $\varepsilon=\alpha /(32 \sqrt{n \log (1 / \beta)})$. Thus $T \varepsilon=\alpha / 8$. We first give a lower bound on the sum. As $\varepsilon^{2} n \leq \alpha$, we have $\left(1-\varepsilon^{2}\right)^{n / 2} \geq 1-n \varepsilon^{2} / 2 \geq 1-\alpha / 2$. Also,

$$
\left(\frac{1+\varepsilon}{1-\varepsilon}\right)^{w t(x)-n / 2} \geq\left(\frac{1-\varepsilon}{1+\varepsilon}\right)^{|w t(x)-n / 2|} \geq\left(\frac{1-\varepsilon}{1+\varepsilon}\right)^{T}=\left(1-\frac{2 \varepsilon}{1+\varepsilon}\right)^{T} \geq 1-2 T \varepsilon=1-\alpha / 4
$$

Therefore,

$$
\begin{aligned}
2^{-n} \sum_{x \in S_{1}} f(x)\left(1-\varepsilon^{2}\right)^{n / 2}\left(\frac{1+\varepsilon}{1-\varepsilon}\right)^{w t(x)-n / 2} & \geq 2^{-n} \sum_{x \in S_{1}} f(x)(1-\alpha / 2)(1-\alpha / 4) \\
& \geq 2^{-n} \sum_{x \in S_{1}} f(x)(1-\alpha) \\
& =\mathrm{E}\left[f\left(X_{n, 0}\right) \mathbb{1}\left(X_{n, 0} \in S_{1}\right)\right](1-\alpha) .
\end{aligned}
$$

Now we bound above the sum. We have

$$
\left(1-\varepsilon^{2}\right)^{n / 2}\left(\frac{1+\varepsilon}{1-\varepsilon}\right)^{w t(x)-n / 2} \leq\left(\frac{1+\varepsilon}{1-\varepsilon}\right)^{T}=\left(1+\frac{2 \varepsilon}{1-\varepsilon}\right)^{T} \leq e^{\frac{2 T \varepsilon}{1-\varepsilon}} \leq e^{4 T \varepsilon}=e^{\alpha / 2} \leq 1+\alpha,
$$

where the last inequality follows from the inequality $e^{x} \leq 1+2 x$ for all $x \in[0,1]$. Hence,

$2^{-n} \sum_{x \in S_{1}} f(x)\left(1-\varepsilon^{2}\right)^{n / 2}\left(\frac{1+\varepsilon}{1-\varepsilon}\right)^{w t(x)-n / 2} \leq 2^{-n} \sum_{x \in S_{1}} f(x)(1+\alpha)=\mathrm{E}\left[f\left(X_{n, 0}\right) \mathbb{1}\left(X_{n, 0} \in S_{1}\right)\right](1+\alpha)$.

Putting the lower and upper bounds together, we have

$$
\begin{aligned}
\mathrm{E}\left[f\left(X_{n, \varepsilon}\right)\right] & =\mathrm{E}\left[f\left(X_{n, \varepsilon}\right) \mathbb{1}\left(X_{n, \varepsilon} \in S_{1}\right)\right]+\mathrm{E}\left[f\left(X_{n, \varepsilon}\right) \mathbb{1}\left(X_{n, \varepsilon} \in S_{2}\right)\right] \\
& =(1 \pm \alpha) \mathrm{E}\left[f\left(X_{n, 0}\right) \mathbb{1}\left(X_{n, 0} \in S_{1}\right)\right]+\mathrm{E}\left[f\left(X_{n, 0}\right) \mathbb{1}\left(X_{n, 0} \in S_{2}\right)\right] \pm \beta \\
& =(1 \pm \alpha)\left(\mathrm{E}\left[f\left(X_{n, 0}\right) \mathbb{1}\left(X_{n, 0} \in S_{1}\right)\right]+\mathrm{E}\left[f\left(X_{n, 0}\right) \mathbb{1}\left(X_{n, 0} \in S_{2}\right)\right]\right) \pm \beta \\
& =(1 \pm \alpha) \mathrm{E}\left[f\left(X_{n, 0}\right)\right] \pm \beta
\end{aligned}
$$

as claimed.

We will apply the following lemma to Claim 4.

Lemma 3.1. Let $k \geq 2$ be an integer and $\alpha \in(0,1 / 2]$ be a real number. For $i \in\{1, \ldots, k\}$, let $a_{i}, a_{i}^{\prime} \in$ $[0,1]$ be any two real numbers such that $a_{i}^{\prime}=a_{i}(1 \pm \alpha) \pm \alpha / k$. We have

$$
\left|\prod_{i \leq k}\left(1-a_{i}\right)-\prod_{i \leq k}\left(1-a_{i}^{\prime}\right)\right| \leq O(\alpha \log (1 / \alpha)) .
$$


Proof. We consider two cases depending on whether $\sum_{i \leq k} a_{i}>2 \log (1 / \alpha)$.

If $\sum_{i \leq k} a_{i}>2 \log (1 / \alpha)$, then as $\alpha \leq 1 / 2$, we have

$\prod_{i \leq k}\left(1-a_{i}^{\prime}\right) \leq e^{-\sum_{i \leq k} a_{i}^{\prime}} \leq e^{-\sum_{i \leq k}\left(a_{i}(1-\alpha)-\alpha / k\right)} \leq e^{-2 \log (1 / \alpha)(1-\alpha)+\alpha} \leq e^{-\log (1 / \alpha)+\alpha} \leq \alpha \cdot e^{\alpha} \leq 2 \alpha$,

and the same bound holds for $\prod_{i \leq k}\left(1-a_{i}\right)$. So $\left|\prod_{i \leq k}\left(1-a_{i}\right)-\prod_{i \leq k}\left(1-a_{i}^{\prime}\right)\right| \leq O(\alpha \log (1 / \alpha))$.

If $\sum_{i \leq k} a_{i} \leq 2 \log (1 / \alpha)$, then as $a_{i}=a_{i}(1 \pm \alpha) \pm \alpha / k$, we have

$$
\begin{aligned}
\prod_{i \leq k}\left(1-a_{i}^{\prime}\right) & =\prod_{i \leq k}\left(1-a_{i}(1 \pm \alpha) \pm \alpha / k\right) \\
& =\prod_{i \leq k}\left(1-a_{i} \pm \alpha a_{i} \pm \alpha / k\right) \\
& =\prod_{i \leq k}\left(1-a_{i}\right) \pm\left(\sum_{i=1}^{k} \sum_{S \subseteq\{1,2, \ldots, k\}:|S|=i} \prod_{j \in S}\left(\alpha a_{j}+\alpha / k\right) \prod_{j \notin S}\left(1-a_{j}\right)\right) \\
& =\prod_{i \leq k}\left(1-a_{i}\right) \pm\left(\sum_{i=1}^{k} \alpha^{i} \sum_{S:|S|=i} \prod_{j \in S}\left(a_{j}+1 / k\right)\right) .
\end{aligned}
$$

So $\left|\prod_{i \leq k}\left(1-a_{i}^{\prime}\right)-\prod_{i \leq k}\left(1-a_{i}\right)\right| \leq \sum_{i=1}^{k} \alpha^{i} S_{i}\left(z_{1}, \ldots, z_{k}\right)$, where

$$
S_{i}\left(z_{1}, \ldots, z_{k}\right)=\sum_{S:|S|=i} \prod_{j \in S} z_{j}, \quad \text { and } \quad z_{j}:=a_{j}+1 / k .
$$

Since each $z_{j} \geq 0$, by Claim 3, we have

$$
S_{i}\left(z_{1}, \ldots, z_{k}\right) \leq(e / i)^{i}\left(\sum_{j \leq k} z_{j}\right)^{i} \leq(e / i)^{i}(2 \log (1 / \alpha)+1)^{i}=(2 e \log (\sqrt{2} / \alpha) / i)^{i},
$$

because $\sum_{j \leq k} a_{j} \leq 2 \log (1 / \alpha)$. Applying the bound to each $S_{i}\left(a_{1}, \ldots, a_{k}\right)$, we have

$$
\left|\prod_{i \leq k}\left(1-a_{i}^{\prime}\right)-\prod_{i \leq k}\left(1-a_{i}\right)\right| \leq \sum_{i=1}^{k}\left(\frac{2 e \alpha \log (\sqrt{2} / \alpha)}{i}\right)^{i}=O(\alpha \log (1 / \alpha)),
$$

because $2 e \alpha \log (\sqrt{2} / \alpha) \leq 1$ for sufficiently small $\alpha$.

We are now ready to prove Theorem 1.1 .

Proof of Theorem 1.1. We look at the bias $\left|\mathrm{E}\left[f_{i}\left(X_{n, 0}\right)\right]\right|$ of each $f_{i}$. There are two cases: If one of them is small, then we argue that both $\left|\mathrm{E}\left[f\left(X_{n k, 0}\right)\right]\right|$ and $\left|\mathrm{E}\left[f\left(X_{n k, \varepsilon}\right)\right]\right|$ are small and so is $\left|\mathrm{E}\left[f\left(X_{n k, 0}\right)\right]-\mathrm{E}\left[f\left(X_{n k, \varepsilon}\right)\right]\right|$. Otherwise, every $f_{i}$ has large bias, and so using Claim 4 we show that both $\mathrm{E}\left[f_{i}\left(X_{n, 0}\right)\right]$ and $\mathrm{E}\left[f_{i}\left(X_{n, \varepsilon}\right)\right]$ have the same sign. In this case, we apply Claim 3.1 to their absolute values.

To proceed, for each $i \in\{1, \ldots, k\}$, define $g_{i}(x):=\left(1-f_{i}(x)\right) / 2 \in[0,1]$. Let $p_{i}$ and $p_{i}^{\prime}$ denote $\mathrm{E}\left[g_{i}\left(X_{n, 0}\right)\right]$ and $\mathrm{E}\left[g_{i}\left(X_{n, \varepsilon}\right)\right]$, respectively. Note that $\mathrm{E}\left[f_{i}\left(X_{n, \varepsilon}\right)\right]=1-2 p_{i}$ and $\mathrm{E}\left[f_{i}\left(X_{n, 0}\right)\right]=1-2 p_{i}^{\prime}$. Applying Claim 4 to $g_{i}$ with $\beta=\alpha / k \leq 1 / 2$, we have $p_{i}^{\prime}=p_{i}(1 \pm \alpha) \pm \alpha / k$. 
Suppose for some $i \in\{1, \ldots, k\},\left|\mathrm{E}\left[f_{i}\left(X_{n, 0}\right)\right]\right| \leq 4 \alpha \leq 1$. Then, by our definition of $g_{i}$, we have $p_{i}=1 / 2 \pm 2 \alpha$. Since $p_{i}^{\prime}=p_{i}(1 \pm \alpha) \pm \alpha / k$, we have

$$
\begin{aligned}
p_{i}^{\prime} & \leq(1 / 2+2 \alpha)(1+\alpha)+\alpha / k \\
& =1 / 2+2 \alpha(1+\alpha)+\alpha / 2+\alpha / k \\
& \leq 1 / 2+3 \alpha+\alpha / 2+\alpha / 2, \\
& =1 / 2+4 \alpha,
\end{aligned}
$$

and

$$
\begin{aligned}
p_{i}^{\prime} & \geq(1 / 2-2 \alpha)(1-\alpha)-\alpha / k \\
& =1 / 2-2 \alpha(1-\alpha)-\alpha / 2-\alpha / k \\
& \geq 1 / 2-2 \alpha-\alpha / 2-\alpha / 2 \\
& =1 / 2-3 \alpha .
\end{aligned}
$$

Hence we have $-3 \alpha \leq \mathrm{E}\left[f_{i}\left(X_{n, \varepsilon}\right)\right] \leq 4 \alpha$. Therefore

$$
\begin{aligned}
\left|\mathrm{E}\left[f\left(X_{n k, 0}\right)\right]-\mathrm{E}\left[f\left(X_{n k, \varepsilon}\right)\right]\right| & \leq\left|\mathrm{E}\left[f\left(X_{n k, 0}\right)\right]\right|+\left|\mathrm{E}\left[f\left(X_{n k, \varepsilon}\right)\right]\right| \\
& \leq\left|\mathrm{E}\left[f_{i}\left(X_{n, 0}\right)\right]\right|+\left|\mathrm{E}\left[f_{i}\left(X_{n, \varepsilon}\right)\right]\right| \\
& \leq 8 \alpha \\
& =O(\alpha \log (1 / \alpha)) .
\end{aligned}
$$

Now we can assume that for all $i \in\{1, \ldots, k\},\left|\mathrm{E}\left[f_{i}\left(X_{n, 0}\right)\right]\right|>4 \alpha$. If $\mathrm{E}\left[f_{i}\left(X_{n, 0}\right)\right]>4 \alpha$, then $p_{i}<$ $1 / 2-2 \alpha$, and

$$
\begin{aligned}
p_{i}^{\prime} & <(1 / 2-2 \alpha)(1+\alpha)+\alpha / k \\
& =1 / 2-2 \alpha(1+\alpha)+\alpha / 2+\alpha / k \\
& \leq 1 / 2-2 \alpha+\alpha / 2+\alpha / 2 \\
& \leq 1 / 2,
\end{aligned}
$$

and hence $\mathrm{E}\left[f_{i}\left(X_{n, \varepsilon}\right)\right] \geq 0$. Otherwise, $\mathrm{E}\left[f_{i}\left(X_{n, 0}\right)\right]<-4 \alpha$ and so $p_{i}>1 / 2+2 \alpha$, then we have

$$
\begin{aligned}
p_{i}^{\prime} & >(1 / 2+2 \alpha)(1-\alpha)-\alpha / k \\
& =1 / 2+2 \alpha(1-\alpha)-\alpha / 2-\alpha / k \\
& \geq 1 / 2+\alpha-\alpha / 2-\alpha / 2 \\
& =1 / 2,
\end{aligned}
$$

and so $\mathrm{E}\left[f_{i}\left(X_{n, \varepsilon}\right)\right] \leq 0$. Hence, we can assume that for every $i \in\{1, \ldots, k\}, \mathrm{E}\left[f_{i}\left(X_{n, 0}\right)\right]$ and $\mathrm{E}\left[f_{i}\left(X_{n, \varepsilon}\right)\right]$ have the same sign, which implies that $\mathrm{E}\left[f\left(X_{n k, 0}\right)\right]$ and $\mathrm{E}\left[f\left(X_{n k, \varepsilon}\right)\right]$ also have the same sign.

Therefore, it suffices to bound above || $\mathrm{E}\left[f\left(X_{n k, 0}\right)\right]|-| \mathrm{E}\left[f\left(X_{n k, \varepsilon}\right)\right] \|$. Let $q_{i}:=1-p_{i}$ and $q_{i}^{\prime}:=$ $1-p_{i}^{\prime}$. Applying Claim 4 to $1-g_{i}$ with $\beta=\alpha / k \leq 1 / 2$, we also have $q_{i}^{\prime}=q_{i}(1 \pm \alpha) \pm \alpha / k$.

Observe that

$$
\left|\mathrm{E}\left[f_{i}\left(X_{n, 0}\right)\right]\right|=1-2 \min \left\{p_{i}, q_{i}\right\} \quad \text { and } \quad\left|\mathrm{E}\left[f_{i}\left(X_{n, \varepsilon}\right)\right]\right|=1-2 \min \left\{p_{i}^{\prime}, q_{i}^{\prime}\right\} .
$$

Thus, if we define $m_{i}:=2 \min \left\{p_{i}, q_{i}\right\}$ and $m_{i}^{\prime}:=2 \min \left\{p_{i}^{\prime}, q_{i}^{\prime}\right\}$, then $\left|\mathrm{E}\left[f_{i}\left(X_{n, 0}\right)\right]\right|=1-m_{i}$ and so $\left|\mathrm{E}\left[f\left(X_{n k, 0}\right)\right]\right|=\prod_{i \leq k}\left|\mathrm{E}\left[f_{i}\left(X_{n, 0}\right)\right]\right|=\prod_{i \leq k}\left(1-m_{i}\right)$. Similarly, we have $\left|\mathrm{E}\left[f\left(X_{n k, \varepsilon}\right)\right]\right|=\prod_{i \leq k}$ 
$\left(1-m_{i}^{\prime}\right)$. Note that $m_{i}, m_{i}^{\prime} \in[0,1]$, and recall that $p_{i}^{\prime}=p_{i}(1 \pm \alpha) \pm \alpha / k$ and $q_{i}^{\prime}=q_{i}(1 \pm \alpha) \pm \alpha / k$, which together imply $m_{i}^{\prime}=m_{i}(1 \pm \alpha) \pm 2 \alpha / k$. Applying Lemma 3.1 to $m_{i}$ and $m_{i}^{\prime}$, we have

$$
\begin{aligned}
\left|\mathrm{E}\left[f\left(X_{n k, 0}\right)\right]-\mathrm{E}\left[f\left(X_{n k, \varepsilon}\right)\right]\right| & =\left\|\mathrm{E}\left[f\left(X_{n k, 0}\right)\right]|-| \mathrm{E}\left[f\left(X_{n k, \varepsilon}\right)\right]\right\| \\
& =\left|\prod_{i \leq k}\left(1-m_{i}\right)-\prod_{i \leq k}\left(1-m_{i}^{\prime}\right)\right| \\
& =O(\alpha \log (1 / \alpha)),
\end{aligned}
$$

proving the claim.

\section{OTHER PROOFS}

In this section, we prove Theorem 1.2 and Claim 1.

Proof of Theorem 1.2. First, note that we can assume $k \in\left[n^{2}, 2^{\alpha n}\right]$ for any desired constant $\alpha>0$, because if $k$ is larger one can make the other functions identically 1 and the expression for $\varepsilon$ does not change.

Let $u^{\prime}$ be the largest integer such that $2^{-n}\left(\begin{array}{c}n \\ n / 2-u^{\prime}\end{array}\right) \geq 1 / k$. For each $i \in\{1, \ldots, k\}$, define $f_{i}$ : $\{0,1\}^{n} \rightarrow\{0,1\}$ to be 1 if and only if $\sum_{j \leq n} x_{j} \neq n / 2-u^{\prime}$. We have

$$
\operatorname{Pr}\left[f\left(X_{n k, 0}\right)=1\right]=\left(1-2^{-n}\left(\begin{array}{c}
n \\
n / 2-u^{\prime}
\end{array}\right)\right)^{k} \leq(1-1 / k)^{k} \leq 1 / e .
$$

We will show that $u^{\prime}=\Theta(1 / \sqrt{n \log k})$, and for $\varepsilon:=1 / u^{\prime}$, we have

$$
\operatorname{Pr}\left[f\left(X_{n, \varepsilon}\right)=1\right] \geq 1-c / k
$$

for a constant $c \in[0,0.45]$. Assuming both, we have

$$
\begin{aligned}
\operatorname{Pr}\left[f\left(X_{n k, 0}\right)=1\right]-\operatorname{Pr}\left[f\left(X_{n k, \varepsilon}\right)=1\right] & \geq(1-c / k)^{k}-1 / e \\
& \geq e^{-2 c}-1 / e \\
& \geq e^{-0.9}-1 / e \\
& \geq 1 / 100,
\end{aligned}
$$

where the second inequality follows from the inequality $1-x \geq e^{-2 x}$ for $x \in[0,1 / 2]$, proving the claim.

It remains to show that $u=\Theta(1 / \sqrt{n \log k})$ and that Inequality (1) holds. Let $H(x):=-x \log x-$ $(1-x) \log (1-x)$ be the binary entropy function. Using the approximations

$$
\frac{2^{n H(t / n)}}{n+1} \leq\left(\begin{array}{l}
n \\
t
\end{array}\right) \leq 2^{n H(t / n)} \text { and } H\left(\frac{1}{2}-\gamma\right)=1-\Theta\left(\gamma^{2}\right),
$$

we have that for every integer $u \geq 0$

$$
2^{-\Theta\left(u^{2} / n\right)-\log (n+1)} \leq 2^{-n}\left(\begin{array}{c}
n \\
n / 2-u
\end{array}\right) \leq 2^{-\Theta\left(u^{2} / n\right)} .
$$

Since $k \geq n^{2}$, from the bounds above we have $u^{\prime}=\sqrt{(n \log k) / c^{\prime}}$ for some $c^{\prime}>0$. 
Now we show Inequality (1). First we establish that $\operatorname{Pr}\left[N_{n, 0}=n / 2-u^{\prime}\right] \in[1 / k, 2 / k]$. The lower bound holds by definition of $u^{\prime}$, and the upper bound is proved next. We have

$$
\begin{aligned}
\operatorname{Pr}\left[N_{n, 0}=n / 2-u^{\prime}\right] & =2^{-n}\left(\begin{array}{c}
n \\
n / 2-u^{\prime}
\end{array}\right) \\
& =2^{-n}\left(\begin{array}{c}
n \\
n / 2-u^{\prime}-1
\end{array}\right)\left(\frac{n-\left(n / 2-u^{\prime}\right)+1}{n / 2-u^{\prime}}\right) \\
& =\operatorname{Pr}\left[N_{n, 0}=n / 2-u^{\prime}-1\right]\left(1+\frac{2 u^{\prime}+1}{n / 2-u^{\prime}}\right) .
\end{aligned}
$$

Since $k \leq 2^{\alpha n}$, we have $u^{\prime}=\sqrt{(n \log k) / c^{\prime}} \leq \sqrt{\alpha / c^{\prime}} \cdot n \leq n / 8$ for a sufficiently small constant $\alpha>$ 0 . Hence,

$$
1+\frac{2 u^{\prime}+1}{n / 2-u^{\prime}} \leq 2
$$

Also, by the definition of $u^{\prime}$, we have $\operatorname{Pr}\left[N_{n, 0}=n / 2-u^{\prime}-1\right]<1 / k$. Therefore,

$$
\operatorname{Pr}\left[N_{n, 0}=n / 2-u^{\prime}\right]=\operatorname{Pr}\left[N_{n, 0}=n / 2-u^{\prime}-1\right]\left(1+\frac{2 u^{\prime}+1}{n / 2-u^{\prime}}\right) \leq 2 / k,
$$

as desired.

Now, by the definition of $f_{i}$,

$$
\begin{aligned}
\operatorname{Pr}\left[f_{i}\left(X_{n, \varepsilon}\right)=0\right] & =2^{-n}\left(\begin{array}{c}
n \\
n / 2-u^{\prime}
\end{array}\right)(1+\varepsilon)^{n / 2-u^{\prime}}(1-\varepsilon)^{n / 2+u^{\prime}} \\
& =\operatorname{Pr}\left[N_{n, 0}=n / 2-u^{\prime}\right]\left(1-\varepsilon^{2}\right)^{n / 2}\left(\frac{1-\varepsilon}{1+\varepsilon}\right)^{u^{\prime}}
\end{aligned}
$$

It follows from

$$
\left(1-\varepsilon^{2}\right)^{n / 2}\left(\frac{1-\varepsilon}{1+\varepsilon}\right)^{u^{\prime}} \leq\left(\frac{1-\varepsilon}{1+\varepsilon}\right)^{u^{\prime}}=\left(1-\frac{2 \varepsilon}{1+\varepsilon}\right)^{u^{\prime}} \leq e^{-\frac{2 \varepsilon u^{\prime}}{1+\varepsilon}}=e^{-\frac{2}{1+\varepsilon}} \leq e^{-1.5}
$$

that $\operatorname{Pr}\left[f_{i}\left(X_{n, \varepsilon}\right)=0\right] \leq(2 / k) \cdot e^{-1.5} \leq 0.446 / k$, showing Inequality (1).

We made no effort to optimize the constants in the above proof, but we point out that by picking $\varepsilon=c / u^{\prime}$ for a constant $c>1$ we can improve the distinguishing advantage from $1 / 100$ to a larger constant.

Proof of Claim 1. For ease of presentation, we will work with inputs over $\{-1,1\}$ instead of $\{0,1\}$, by translating each input bit $x$ by $1-2 x$. Hence, each $X_{i}$ equals -1 with probability $(1+\varepsilon) / 2$ and 1 with probability $(1-\varepsilon) / 2$. We also use the random variable $N_{n, \varepsilon}$ to denote the sum of $n$ independent $X_{i}$ 's. Note that in this case we have $\mathrm{E}\left[N_{n, \varepsilon}\right]=-\varepsilon n$.

Let $m:=n k$. The idea is to rotate along the complex unit circle with respect to the number of -1 's in the input $x$, so that if the number of -1 's in $x$ is close to $m / 2$ we are close to the point 1 , whereas if it is close to $(1+\varepsilon) m / 2$, we are close to the point -1 . Then the claim follows from Hoeffding's inequality.

Specifically, for each $\ell \in\{1, \ldots, k\}$, define $f_{\ell}:\{0,1\}^{n} \rightarrow \mathbb{C}_{=1}$ to be $f_{\ell}(x):=e^{i \frac{\pi}{\varepsilon m} \sum_{j \leq n} x_{j}}$. Note that

$$
f\left(x_{1}, \ldots, x_{k}\right)=\prod_{\ell \leq k} f_{\ell}\left(x_{\ell}\right)=e^{i \frac{\pi}{\varepsilon m} \sum_{j \leq m} x_{j}} .
$$

Observe that when $\sum_{j \leq m} x_{j}$ is close to $0=\mathrm{E}\left[N_{m, 0}\right], f(x)$ is close to 1 , whereas when $\sum_{j \leq m} x_{j}$ is close to $-\varepsilon m=\mathrm{E}\left[N_{m, \varepsilon}\right], f(x)$ is close to -1 . By Hoeffding's inequality, we have for $\tau=0$ and $\tau=\varepsilon$,

$$
\operatorname{Pr}\left[\left|N_{m, \tau}-\tau m\right|>\varepsilon m / 3\right] \leq 2 e^{-\Omega\left(\varepsilon^{2} m\right)} .
$$


Conditioned on the events $\left|N_{m, \tau}-\tau m\right| \leq \varepsilon m / 2$ for both $\tau=0$ and $\tau=\varepsilon$, we have $f\left(X_{m, \tau}\right)=$ $e^{i \pi \tau / \varepsilon} \cdot e^{i \pi \theta}$ for some $\theta \in[-0.5,0.5]$. Hence,

$$
\left|f\left(X_{m, 0}\right)-f\left(X_{m, \varepsilon}\right)\right|=\left|1 \cdot e^{i \pi \theta}-(-1) \cdot e^{i \pi \theta^{\prime}}\right|=\left|e^{i \pi \theta}+e^{i \pi \theta^{\prime}}\right|
$$

for some $\theta, \theta^{\prime} \in[-0.5,0.5]$, which is at least

$$
\min _{\theta, \theta^{\prime} \in[-0.5,0.5]}\left|e^{i \pi \theta}+e^{i \pi \theta^{\prime}}\right| \geq \min _{\theta, \theta^{\prime} \in[-0.5,0.5]} \mathcal{R}\left(e^{i \pi \theta}+e^{i \pi \theta^{\prime}}\right)=2 \min _{\theta \in[-0.5,0.5]} \cos \theta \geq 2 \cos (0.5) \geq 1.5,
$$

where $\mathfrak{R}$ denotes the real part and the last inequality follows from the inequality $\cos (\alpha) \geq 1-\alpha^{2}$. Therefore,

$$
\begin{aligned}
\left|\mathrm{E}\left[f\left(X_{m, 0}\right)\right]-\mathrm{E}\left[f\left(X_{m, \varepsilon}\right)\right]\right| & \geq\left(1-2 e^{-\Omega\left(\varepsilon^{2} m\right)}\right) \cdot 1.5 \\
& =1.5-e^{-\Omega\left(\varepsilon^{2} m\right)},
\end{aligned}
$$

as desired.

To provide a slightly more complete picture of the effect of changing range on the distinguishing advantage, we include the following claim that shows that, for a single function, range $\mathbb{C}_{=1}$ and range $\{0,1\}$ are equivalent.

Claim 5. Let $X$ and $Y$ be two discrete random variables over the same support $Z$. We have

$$
\max _{f: Z \rightarrow \mathbb{C}_{=1}}|\mathrm{E}[f(X)]-\mathrm{E}[f(Y)]|=2 \max _{g: Z \rightarrow\{0,1\}}|\mathrm{E}[g(X)]-\mathrm{E}[g(Y)]| .
$$

Proof. Let $p$ and $q$ be the probability mass functions of $X$ and $Y$, respectively. We will use the well-known fact that

$$
2 \max _{g: Z \rightarrow\{0,1\}}|\mathrm{E}[g(X)]-\mathrm{E}[g(Y)]|=\sum_{z \in Z}|p(z)-q(z)|
$$

and work with the quantity on the right-hand side.

For any $f: Z \rightarrow \mathbb{C}_{=1}$ we have

$$
\begin{aligned}
|\mathrm{E}[f(X)]-\mathrm{E}[f(Y)]| & =\left|\sum_{z \in Z} p(z) f(z)-q(z) f(z)\right| \\
& =\left|\sum_{z \in Z}(p(z)-q(z)) f(z)\right| \\
& \leq \sum_{z \in Z}|p(z)-q(z)||f(z)| \\
& \leq \sum_{z \in Z}|p(z)-q(z)| .
\end{aligned}
$$

On the other hand, define $f: Z \rightarrow \mathbb{C}_{=1}$ to be $f(z)=1$ if $p(z) \geq q(z)$ and -1 otherwise. We have

$$
\begin{aligned}
\sum_{z \in Z}|p(z)-q(z)| & =\sum_{z: p(z) \geq q(z)}(p(z)-q(z))+\sum_{z: p(z)<q(z)}(q(z)-p(z)) \\
& =\sum_{z \in Z}(p(z)-q(z)) f(z) \\
& =\mathrm{E}[f(X)]-\mathrm{E}[f(Y)] .
\end{aligned}
$$

The claim follows. 


\section{ACKNOWLEDGMENTS}

We thank the referees for the useful feedback, and in particular for raising the question of extending Theorem 1.1 in a preliminary version of this article to functions with range $[-1,1]$.

\section{REFERENCES}

[1] Scott Aaronson. 2010. BQP and the polynomial hierarchy. In Proceedings of the 42nd ACM Symposium on the Theory of Computing (STOC'10). ACM, 141-150.

[2] Scott Aaronson and Andrew Drucker. 2011. Advice coins for classical and quantum computation. In Automata, Languages and Programming. Part I. Lecture Notes in Computer Science, Vol. 6755. Springer, Heidelberg, 61-72. https://doi.org/10.1007/978-3-642-22006-7_6

[3] Miklós Ajtai. 1983. $\Sigma_{1}^{1}$-formulae on finite structures. Ann. Pure Appl. Logic 24, 1 (1983), 1-48.

[4] Miklós Ajtai, János Komlós, and Endre Szemerédi. 1987. Deterministic simulation in LOGSPACE. In Proceedings of the 19th ACM Symposium on the Theory of Computing (STOC'87). 132-140.

[5] Roy Armoni, Michael E. Saks, Avi Wigderson, and Shiyu Zhou. 1996. Discrepancy sets and pseudorandom generators for combinatorial rectangles. In Proceedings of the 37th IEEE Symposium on Foundations of Computer Science (FOCS'96). 412-421.

[6] Joshua Brody and Elad Verbin. 2010. The coin problem, and pseudorandomness for branching programs. In Proceedings of the 51th IEEE Symposium on Foundations of Computer Science (FOCS'10).

[7] Gil Cohen, Ivan Bjerre Damgård, Yuval Ishai, Jonas Kölker, Peter Bro Miltersen, Ran Raz, and Ron D. Rothblum. 2013. Efficient multiparty protocols via log-depth threshold formulae (extended abstract). In Proceedings of the International Cryptology Conference (CRYPTO'13). 185-202. DOI : http://dx.doi.org/10.1007/978-3-642-40084-1_11

[8] Gil Cohen, Anat Ganor, and Ran Raz. 2014. Two sides of the coin problem. In Proceedings of the Workshop on Randomization and Computation (RANDOM'14). 618-629. DOI : http://dx.doi.org/10.4230/LIPIcs.APPROX-RANDOM.2014.618

[9] Guy Even, Oded Goldreich, Michael Luby, Noam Nisan, and Boban Velickovic. 1998. Efficient approximation of product distributions. Rand. Struct. Algor. 13, 1 (1998), 1-16.

[10] Parikshit Gopalan, Daniel Kane, and Raghu Meka. 2015. Pseudorandomness via the discrete fourier transform. In Proceedings of the IEEE Symposium on Foundations of Computer Science (FOCS'15). 903-922. DOI : http://dx.doi.org/10. 1109/FOCS.2015.60

[11] Parikshit Gopalan, Raghu Meka, Omer Reingold, Luca Trevisan, and Salil Vadhan. 2012. Better pseudorandom generators from milder pseudorandom restrictions. In Proceedings of the IEEE Symposium on Foundations of Computer Science (FOCS'12).

[12] Parikshit Gopalan and Amir Yehudayoff. 2014. Inequalities and tail bounds for elementary symmetric polynomials. Electronic Colloquium on Computational Complexity (ECCC) 21 (2014), 19. http://eccc.hpi-web.de/report/2014/019.

[13] Elad Haramaty, Chin Ho Lee, and Emanuele Viola. 2017. Bounded independence plus noise fools products. In Proceedings of the Conference on Computational Complexity (CCC'17).

[14] Martin E. Hellman and Thomas M. Cover. 1970. Learning with finite memory. Ann. Math. Statist. 41 (1970), 765-782. https://doi.org/10.1214/aoms/1177696958

[15] Russell Impagliazzo, Noam Nisan, and Avi Wigderson. 1994. Pseudorandomness for network algorithms. In Proceedings of the 26th ACM Symposium on the Theory of Computing (STOC'94). 356-364.

[16] Chi-Jen Lu. 2002. Improved pseudorandom generators for combinatorial rectangles. Combinatorica 22, 3 (2002), 417433.

[17] Noam Nisan. 1992. Pseudorandom generators for space-bounded computation. Combinatorica 12, 4 (1992), $449-461$.

[18] Noam Nisan and David Zuckerman. 1996. Randomness is linear in space. f. Comput. Syst. Sci. 52, 1 (Feb. 1996), 43-52.

[19] Ronen Shaltiel and Emanuele Viola. 2010. Hardness amplification proofs require majority. SIAM f. Comput. 39, 7 (2010), 3122-3154.

[20] J. Michael Steele. 2004. The Cauchy-Schwarz Master Class. Mathematical Association of America, Washington, DC. DOI : http://dx.doi.org/10.1017/CBO9780511817106

[21] John P. Steinberger. 2013. The distinguishability of product distributions by read-once branching programs. In Proceedings of the IEEE Conference on Computational Complexity (CCC'13). 248-254. DOI : http://dx.doi.org/10.1109/CCC. 2013.33

[22] Leslie G. Valiant. 1984. Short monotone formulae for the majority function. J. Algor. 5, 3 (1984), 363-366.

[23] Emanuele Viola. 2014. Randomness buys depth for approximate counting. Comput. Complex. 23, 3 (2014), 479-508.

[24] Thomas Watson. 2013. Pseudorandom generators for combinatorial checkerboards. Comput. Complex. 22, 4 (2013), 727-769. DOI : http://dx.doi.org/10.1007/s00037-012-0036-6

Received May 2017; revised January 2018; accepted February 2018 